\title{
Crosstalk between FLS and chondrocytes is regulated by HIF-2 $\alpha$-mediated cytokines in arthritis
}

\author{
Yun Hyun Huh${ }^{1}$, Gyuseok Lee ${ }^{2}$, Won-Hyun Song ${ }^{3}$, Jeong-Tae Koh ${ }^{3,4}$ and Je-Hwang Ryu ${ }^{3,4}$
}

Rheumatoid arthritis (RA) and osteoarthritis (OA), two common types of arthritis, affect the joints mainly by targeting the synovium and cartilage. Increasing evidence indicates that a significant network connects synovitis and cartilage destruction during the progression of arthritis. We recently demonstrated that hypoxia-inducible factor (HIF)-2 $\alpha$ causes RA and OA by regulating the expression of catabolic factors in fibroblast-like synoviocytes (FLS) or chondrocytes. To address the reciprocal influences of HIF-2 $\alpha$ on FLS and chondrocytes, we applied an in vitro co-culture system using a transwell apparatus. When co-cultured with HIF-2 $\alpha$-overexpressing chondrocytes, FLS exhibited increased expression of matrix metalloproteinases and inflammatory mediators, similar to the effects induced by tumor-necrosis factor (TNF)- $\alpha$ treatment of FLS. Moreover, chondrocytes co-cultured with HIF-2 $\alpha$-overexpressing FLS exhibited upregulation of Mmp3 and Mmp13, which is similar to the effects induced by interleukin (IL)- 6 treatment of chondrocytes. We confirmed these differential HIF-2 $\alpha$-induced effects via distinct secretory mediators using 116 -knockout cells and a TNF- $\alpha$-blocking antibody. The FLS-co-culture-induced gene expression changes in chondrocytes were significantly abrogated by IL- 6 deficiency, whereas TNF- $\alpha$ neutralization blocked the alterations in gene expression associated with co-culture of FLS with chondrocytes. Our results further suggested that the observed changes might reflect the HIF-2 $\alpha$-induced upregulation of specific receptors for TNF- $\alpha$ (in FLS) and IL-6 (in chondrocytes). This study broadens our understanding of the possible regulatory mechanisms underlying the crosstalk between the synovium and cartilage in the presence of HIF-2 $\alpha$, and may suggest potential new anti-arthritis therapies. Experimental \& Molecular Medicine (2015) 47, e197; doi:10.1038/emm.2015.88; published online 4 December 2015

\section{INTRODUCTION}

Rheumatoid arthritis (RA) is a polyarticular chronic inflammatory autoimmune disease that mainly affects the joints, where it is associated with tissue destruction and functional disability. ${ }^{1}$ A synovitis involving infiltration of mononuclear cells into the synovial membrane is a characteristic of RA. ${ }^{2}$ Activated fibroblast-like synoviocytes (FLS), which are among the key players in RA joint destruction, are responsible for initiating, propagating and maintaining synovial inflammation by secreting proinflammatory mediators, including interleukin (IL)-1 $\beta$, IL-6, tumor-necrosis factor (TNF)- $\alpha$ and chemokines. ${ }^{3}$ During RA, cartilage can be destroyed by synovium-derived inflammatory mediators (for example, IL-1 $\beta$ and IL-6) that upregulate matrix-degrading enzymes. ${ }^{3}$ In contrast to RA, osteoarthritis (OA) has typically been considered a prototypical non-inflammatory degenerative disease, largely because its major target tissue (articular cartilage) is avascular and aneural.
However, recent studies have focused on the involvement of inflammatory components (for example, joint pain, swelling, stiffness and indicators of synovitis) in the pathology of OA. ${ }^{4}$ Moreover, inflammatory cytokines, chemokines and other inflammatory mediators (for example, inducible nitric oxide synthase, cyclooxygenase- 2 and several matrix metalloproteinases) are produced by chondrocytes, and can be measured in the synovial fluids of OA patients. ${ }^{4}$ Synovitis is reportedly common in both early and late stages of human clinical OA pathology, ${ }^{5}$ and proinflammatory mediators, matrix metalloproteinases $s$ and IL- 6 can be detected in OA synovial fluid samples, although at significantly lower levels than those found in RA patients. ${ }^{6}$ Chondrocytes, which are the sole resident cells of adult human articular cartilage, synthesize cartilage-specific extracellular matrix components under normal low-turnover conditions and in response to various catabolic and anabolic factors associated with the pathogeneses of $\mathrm{OA}$ and RA.

\footnotetext{
${ }^{1}$ Bio Imaging and Cell Dynamics Research Center, Gwangju Institute of Science and Technology, Gwangju, Republic of Korea; ${ }^{2}$ Cell Dynamics Research Center and School of Life Sciences, Gwangju Institute of Science and Technology, Gwangju, Republic of Korea; ${ }^{3}$ Research Center for Biomineralization Disorders and Dental Science Research Institute, School of Dentistry, Chonnam National University, Gwangju, Republic of Korea and ${ }^{4}$ Department of Pharmacology and Dental Therapeutics, School of Dentistry, Chonnam National University, Gwangju, Republic of Korea Correspondence: Professor J-H Ryu, Department of Pharmacology and Dental Therapeutics, School of Dentistry, Chonnam National University, Gwangju 500-757, Republic of Korea.

E-mail: jesryu@jnu.ac.kr
}

Received 4 August 2015; revised 3 September 2015; accepted 14 September 2015 
Although clear understanding of cellular and molecular interactions occuring in synovial tissues is necessary for the pathogenesis of joint inflammation and cartilage destruction in RA and OA, a crosstalk of chondrocytes and FLS still not yet veiled.

Hypoxia-inducible factor (HIF)-2 $\alpha$ (also designated endothelial PAS domain protein-1 or Epas1) is a member of the basic helix-loop-helix/PAS transcription factor family ${ }^{7,8}$ and is known to be regulated by oxygen tension. ${ }^{7}$ HIF- $2 \alpha$ acts as an essential catabolic regulator in the hypoxic inflamed synovium ${ }^{9}$ during the pathogeneses of $\mathrm{OA}^{10}$ and $\mathrm{RA}^{11}$ by modulating the expression levels of various catabolic factors, including $M m p s$, nitric oxide synthase 2 (Nos2; encoding inducible nitric oxide synthase) and prostaglandin-endoperoxide synthase 2 (Ptgs2; encoding cyclooxygenase -2 ), in both chondrocytes and FLS. We previously showed that HIF- $2 \alpha$ also upregulates the expression levels of various secreted mediators, including the cytokines, IL- 6 and TNF- $\alpha$, and the chemokines, CCL5 and CXCL2. ${ }^{10,11}$ It is well accepted that chondrocytes, synoviocytes and infiltrating macrophages can produce inflammatory mediators (cytokines and/or chemokines) and also be activated by these mediators, suggesting that joint tissues may harbor interacting regulatory networks of inflammatory and secretory mediators. ${ }^{12}$ In this study, we used an in vitro co-culture system to broaden our understanding of the role and underlying molecular mechanisms of HIF- $2 \alpha$ in arthritis (especially regarding the association between synovial inflammation and cartilage destruction) and further characterized a major mediator (HIF-2 $\alpha$-upregulated cytokines) involved in the HIF- $2 \alpha$ induced crosstalk between FLS and chondrocytes.

\section{MATERIALS AND METHODS}

\section{Mice and experimental arthritis}

Male DBA/1 J and C57BL/6 mice were bred and maintained in specific pathogen-free barrier facilities. All mouse experiments were conducted in accordance with protocols approved by the Animal Care and Ethics Committees of Chonnam National University. For the experimental model of RA, collagen-induced arthritis was induced in DBA/1 J mice using the previously described protocol. ${ }^{11}$ For the experimental model of arthritic cartilage destruction, C57BL/6 mice were subjected to intra-articular injection of Epas1 adenovirus (Ad-Epas1), as described previously. ${ }^{10}$ In brief, Ad-Epas $1\left(1 \times 10^{9}\right.$ plaque-forming units in a total volume of $10 \mu \mathrm{l}$ ) were injected by Hamilton syringe once a week for 3 weeks into the knee articular joint of 8- to 10-week-old male mice. Empty adenovirus (Ad-C) was used as a control. Mice were killed 3 weeks after the first intra-articular injection for histological and biochemical analyses. Experimental OA was induced in C57BL/6 mice by destabilization of medial meniscus surgery, as described previously. ${ }^{10,13}$ Sham operations were used as controls. Eight weeks later, knee joints were processed for histological analysis.

\section{Histological analysis of joint tissue and immunostaining}

Mouse joint tissues were fixed with $4 \%$ fresh paraformaldehyde for $24 \mathrm{~h}$, decalcified with $0.5 \mathrm{~m}$ ethylenediaminetetraacetic acid ( $\mathrm{pH} 8.0$ ) for 2 weeks, embedded in paraffin and sectioned at $5-\mu \mathrm{m}$ thickness. Joint sections were subjected to safranin-O/hematoxylin staining, as previously described. ${ }^{10,11}$ Cartilage destruction was scored by the modified Mankin's scoring method (grades 0-14), wherein joint tissues were scored for structure ( $0-6$ points), cellularity ( $0-3$ points), matrix staining ( $0-4$ points) and tidemark integrity (0-1 points). ${ }^{14}$ Synovial inflammation (grade 0-4) was scored as previously described: ${ }^{11}$ grade 0 , no sign of inflammation; grade 1 , hyperplasia of the synovial lining without cartilage destruction; and grades 2-4, increasing degrees of immune cell infiltration and cartilage/bone destruction. ${ }^{15}$

\section{Mouse FLS and articular chondrocyte culture}

FLS were isolated from the joint synovium of wild-type and Il6-knockout $\left(I l 6^{-/-}\right)$mice, as described previously. ${ }^{11}$ Subcultured FLS (between passages 4-8) were used for experiments. Flow cytometry was performed with antibodies against CD90 (a fibroblast marker) and CD14 (a macrophage marker) (Abcam, Cambridge, UK) to confirm the purity of our FLS ( $>90 \%$ CD $90+/<1 \%$ CD14+). For isolation of chondrocytes, mouse cartilage tissues were isolated from the femoral condyles and tibial plateaus of wild-type and $I l 6^{-1-}$ mice, and chondrocytes were extracted by digestion with $0.2 \%$ collagenase type II, as described previously. ${ }^{10,16}$ Chondrocytes or FLS were treated with the indicated amounts of recombinant mouse IL-6 protein (MerckMillipore, Billerica, MA, USA), recombinant mouse TNF- $\alpha$ protein (Merck-Millipore) or 800 MOI (multiplicity of infection) of empty adenovirus (Ad-C) or HIF-2 $\alpha$-expressing adenovirus (Ad-Epas1) for $24 \mathrm{~h}$ with or without subsequent treatment with the anti-TNF- $\alpha$ blocking antibody (R\&D Systems Inc., Minneapolis, MN, USA).

\section{Co-culture of mouse FLS and articular chondrocytes}

Chondrocytes and FLS from wild-type or $I l 6^{-/-}$mice were co-cultured in a transwell plate (24-well type; Sigma-Aldrich, St Louis, MO, USA) with a cell culture insert $(0.4-\mu \mathrm{m}$ pore size; SigmaAldrich), which not only physically separated the chondrocytes and FLS but also allowed their secreted factors to pass through. FLS or chondrocytes were grown for 2 days in the transwell plate and washed three times with PBS. Simultaneously, the opposite cell type was seeded on the upper layer of the insert, grown for 2 days, and infected with 800 MOI of Ad-Epas1 for $2 \mathrm{~h}$. The cell culture inserts were then placed in transwell plates, and the cells were co-cultured for an additional $24 \mathrm{~h}$. The cells in the transwell plates were harvested for further analysis.

RNA isolation, reverse transcription-polymerase chain reaction (RT-PCR) and quantitative real time-PCR (qRT-PCR) Total RNA samples from mouse articular chondrocytes and FLS were isolated using the TRI reagent (MRC, Cincinnati, OH, USA), and complementary DNA was synthesized by reverse transcription and subjected to PCR. Relative transcript levels were measured by qRT-PCR using SYBR Premix Ex Taq (TaKaRa Bio Inc., Shiga, Japan). All qRT-PCR reactions were performed in duplicate, and the threshold cycle value of each target gene was normalized with respect to that of glyceraldehyde-3-phosphate dehydrogenase (Gapdh). All of the utilized PCR primers are summarized in Table 1.

\section{Enzyme-linked immunosorbent assay and neutralization of TNF- $\alpha$}

Mouse articular chondrocytes and FLS were infected with empty adenovirus or Ad-Epas1 and incubated in serum-free medium (Dulbecco's Modified Eagle's medium) for $24 \mathrm{~h}$. The concentrations of IL- 6 and TNF- $\alpha$ secreted to the culture media were measured by enzyme-linked immunosorbent assay (Thermo Fisher Scientific Inc., Waltham, MA, USA). For neutralization of TNF- $\alpha$ in co-culture, 
Table 1 PCR primers and conditions

\begin{tabular}{|c|c|c|c|c|c|}
\hline Gene & Strand & Primer sequences & Size & At & Origin \\
\hline Adamts4 & As & 5'-GCCCATCATCTTCCACAATAG-3' & & & \\
\hline Adamts 5 & As & 5'-TCAGTCCCATCCGTAACCTTTG-3' & & & \\
\hline \multirow[t]{2}{*}{ Epas1 } & S & 5'-CGAGAAGAACGACGTGGTGTTC-3' & 370 bp & $62^{\circ} \mathrm{C}$ & Mouse \\
\hline & As & 5'-GTGAAGGCGGGCAGGCTCC-3' & & & \\
\hline Gapdh & As & 5'-TGTAGGCCATGAGGTCCAC-3' & & & \\
\hline \multirow[t]{2}{*}{116} & $\mathrm{~S}$ & 5'-ACCACTCCCAACAGACCTGTCTATACC-3' & $435 \mathrm{bp}$ & $60^{\circ} \mathrm{C}$ & Mouse \\
\hline & As & 5'-CTCCTTCTGTGACTCCAGCTTATCTGTTAG-3' & & & \\
\hline \multirow[t]{2}{*}{ Nos2 } & $\mathrm{S}$ & 5'-TCACTGGGACAGCACAGAAT-3' & $510 \mathrm{bp}$ & $62^{\circ} \mathrm{C}$ & Mouse \\
\hline & As & 5'-TGTGTCTGCAGATGTGCTGA-3' & & & \\
\hline Mmp2 & $\mathrm{S}$ & 5'-CCAACTACGATGATGAC-3' & 233 bp & $60^{\circ} \mathrm{C}$ & Mouse \\
\hline \multirow[t]{2}{*}{ Mmp9 } & $\mathrm{S}$ & 5'-ACCACATCGAACTTCGA-3' & $212 b p$ & $58^{\circ} \mathrm{C}$ & Mouse \\
\hline & As & 5'-CGACCATACAGATACTG-3' & & & \\
\hline \multirow[t]{2}{*}{ Mmp12 } & S & 5'-CCCAGAGGTCAAGATGGATG-3' & 482 bp & $60^{\circ} \mathrm{C}$ & Mouse \\
\hline & As & 5'-GGCTCCATAGAGGGACTGAA-3' & & & \\
\hline \multirow[t]{2}{*}{ Mmp13 } & $\mathrm{S}$ & 5'-TGATGGACCTTCTGGTCTTCTGG-3' & 473 bp & $55^{\circ} \mathrm{C}$ & Mouse \\
\hline & As & 5'-CATCCACATGGTTGGGAAGTTCT-3' & & & \\
\hline \multirow[t]{2}{*}{ Mmp14 } & $\mathrm{S}$ & 5'-GTGCCCTAGGCCTACATCCG-3' & 580 bp & $55^{\circ} \mathrm{C}$ & Mouse \\
\hline & As & 5'-TTGGGTATCCATCCATCACT-3' & & & \\
\hline \multirow[t]{2}{*}{ Mmp15 } & $\mathrm{S}$ & 5'-GAGAGATGTTTGTGTTCAAGGG-3' & $260 \mathrm{bp}$ & $55^{\circ} \mathrm{C}$ & Mouse \\
\hline & As & 5'-TGTGTCAATGCGGTCATAGGG-3' & & & \\
\hline Ptgs2 & $\mathrm{S}$ & 5'-GGTCTGGTGCCTGGTCTGATGAT-3' & 724 bp & $65^{\circ} \mathrm{C}$ & Mouse \\
\hline
\end{tabular}

Abbreviations: As, antisense primer; AT, annealing temperature; $\mathrm{S}$, sense primer.

mouse chondrocytes and FLS grown in cell culture inserts were infected with $800 \mathrm{MOI}$ of Ad-Epas 1 for $12 \mathrm{~h}$, the inserts were placed in transwell plates seeded with the other cell type, and the cells were coincubated in serum-free Dulbecco's Modified Eagle's medium with anti-immunoglobulin $\mathrm{G}(1 \mu \mathrm{g})$ or anti-TNF- $\alpha(0.25,0.5,1.0 \mu \mathrm{g})$ antibodies (R\&D Systems Inc.) for an additional $12 \mathrm{~h}$. The cells in the transwell plates were harvested for further analysis.

\section{Statistical analysis}

The non-parametric Mann-Whitney $U$-test was used to analyze the ordinal grades (for example, synovitis and Mankin scores). Data obtained with qRT-PCR assays were initially tested for conformation to normal distribution using the Shapiro-Wilk test and subsequently analyzed with the Student's $t$-test (pair-wise comparisons) or analysis of variance with post hoc tests (multi-comparison), as appropriate. Significance was accepted at the 0.05 level of probability $(P<0.05)$.

\section{RESULTS}

Synovitis and cartilage destruction are closely related during RA and OA

In an attempt to gain new insights into the possible links and related regulatory mechanisms between the synovium and cartilage in an arthritic joint, we first examined the co-occurrence of these phenotypes in mouse models of experimentally induced arthritis. RA, which is primarily characterized by synovial hyperplasia and synovitis leading to cartilage destruction, was induced using collagen-induced arthritis. As shown in Figure 1a, safranin-O/hematoxylin staining revealed co-occurrence of severe synovitis and cartilage destruction in collagen-induced arthritis joints compared with nonimmunized control joints. The severity of synovial inflammation was scored from 0 to 4 , whereas cartilage destruction was scored from 1 to 14 using Mankin's method. OA, another major type of arthritis, was induced by destabilization of medial meniscus surgery and the joint was stained with safranin-O/ hematoxylin. OA, which is known as non-inflammatory arthritis, is characterized by the erosion of cartilage via the chondrocytic upregulation of matrix-degrading enzymes. To elucidate whether the phenotypic changes of the synovium in this model are followed by cartilage destruction, the OA joints were subdivided into stages of degeneration based on the severity of cartilage damage, and then assessed for the degree of synovitis. Some joint tissues with severe cartilage damage (Mankin's score $>9$ ) showed mild synovitis (scores of 

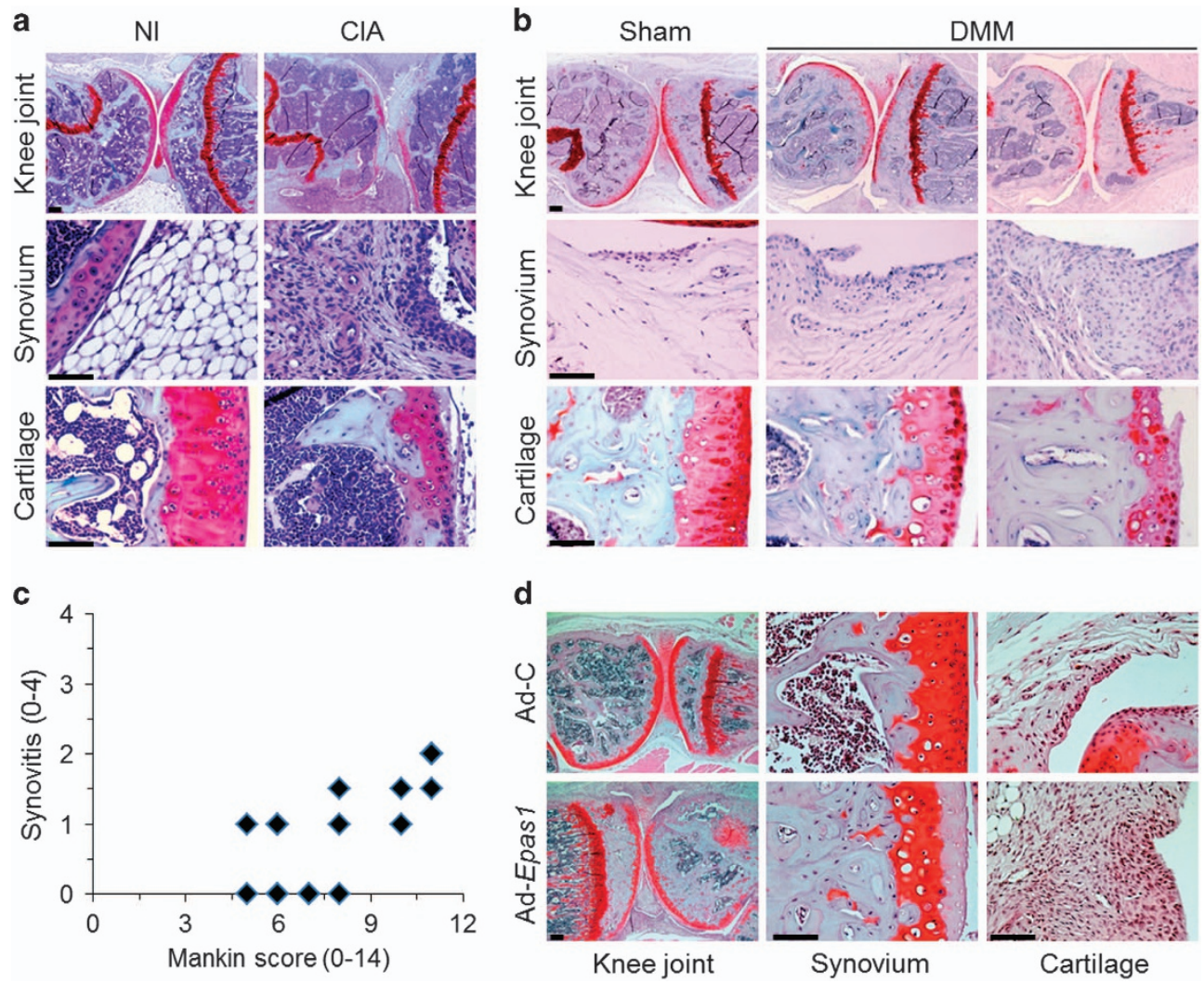

Figure 1 Co-occurrence of synovial inflammation and cartilage erosion in experimental RA and OA mouse models. (a) Safranin-O/ hematoxylin staining and enlarged images of synovium and cartilage in knee joints of DBA/1 J mice immunized with type II collagen (CIA) or not immunized ( $\mathrm{NI}$ ) ( $n=15$ per group). (b) Representative images of synovium and cartilage in knee joints from C57BL/6 mice after sham surgery or destabilization of the medial meniscus (DMM) ( $n=15$ per group). (c) Scoring of synovial inflammation (synovitis) or cartilage destruction (Mankin score) in DMM surgery-induced OA mice. (d) Safranin-O/hematoxylin staining in the cartilage of C57BL/6 mice injected with empty adenovirus $(\mathrm{Ad}-\mathrm{C})$ or Ad-Epas $1\left(1 \times 10^{9}\right.$ plaque-forming units) ( $n=10$ per group). Scale bar: $50 \mu \mathrm{m}$.

$0.5 \sim 2.0$ ) (Figure $1 \mathrm{~b}$ ), and the severity of cartilage erosion and synovitis tended to correlate (Figure 1c). These results suggest that crosstalk may occur between the synovium and the cartilage during experimentally induced RA and OA. We previously showed that HIF-2 $\alpha$ (encoded by Epas1) is an essential catabolic regulator of $\mathrm{RA}^{11}$ and $\mathrm{OA}^{10}$, and that it acts by modulating the expression levels of various catabolic factors in FLS and chondrocytes, respectively. In the present system, we found that overexpression HIF- $2 \alpha$ by injection of Ad-Epas1 into the joint resulted in simultaneous and severe synovitis and cartilage erosion, similar to our observations in RA and OA joints (Figure 1d). Therefore, to further investigate the potential links between FLS and chondrocytes, we examined the secretory proteins induced by HIF- $2 \alpha$.

\section{Altered gene expression in FLS co-cultured with HIF-2 $\alpha$ - overexpressing chondrocytes, and vice versa}

Chondrocytes induced to overexpress HIF-2 $\alpha$ by infection with Ad-Epas1 showed significantly increased mRNA levels of matrix-degrading enzymes (Mmp3, Mmp9, Mmp12, Mmp13 and Adamts4), inflammatory mediators (Ptgs2 and Nos2) and proinflammatory cytokines (Il6 and Tnfa) (Figure 2a). To address reciprocal influences between chondrocytes and FLS by HIF-2 $\alpha$, we applied a co-culture system using transwell plates with a pore diameter of $0.4 \mu \mathrm{m}$, which enabled the transfer of secretory molecules but not cells. To examine how secretory mediators originating from HIF- $2 \alpha$-overexpressing chondrocytes affected FLS, we cultured primary FLS from healthy mice on the bottom of the apparatus and Ad-Epas1-infected chondrocytes on the membrane of the insert. Interestingly, RT-PCR showed that the expression levels of Mmp3, Mmp9, Mmp12, Mmp13, Adamts4, Ptgs2 and Nos2 was increased in co-cultured FLS, even though HIF- $2 \alpha$ expression was not altered in these cells (Figure 2b). This observation was confirmed and quantified by qRT-PCR (Figure 2c).

Next, we performed the reciprocal experiment. HIF-2 $\alpha$ overexpressing FLS showed exactly same pattern of upregulated catabolic factors by chondrocytes (Figure 3a). Among the examined cytokines (Il1 $\beta, I l 6, I l 11, I l 12, I l 17, I l 21$, Lif and Tnfa; data not shown), Il6 and Tnfa were increased by overexpression of HIF-2 $\alpha$ in FLS (Figure 3a). Interestingly, chondrocytes co-cultured with Ad-Epas1-infected FLS showed increased expression levels of Mmp3, Mmp13 and Nos2, as 
a

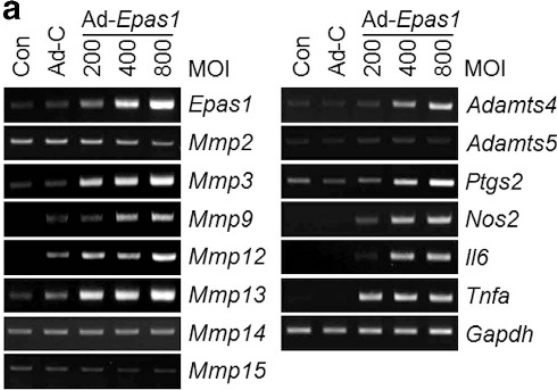

Mono-cultured chondrocytes b

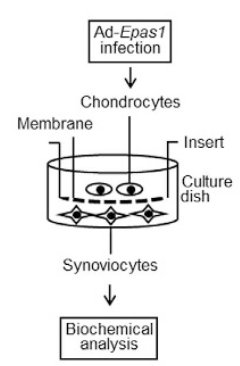

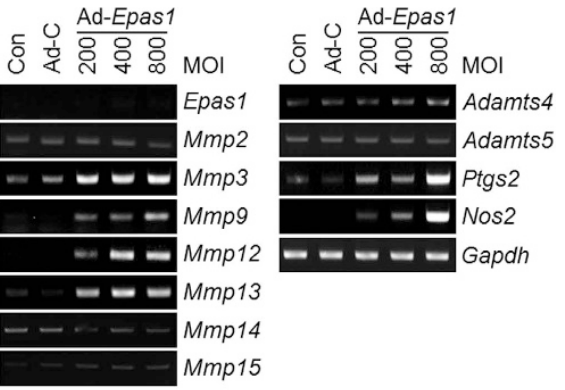

Co-cultured FLS w/ chondrocytes

c
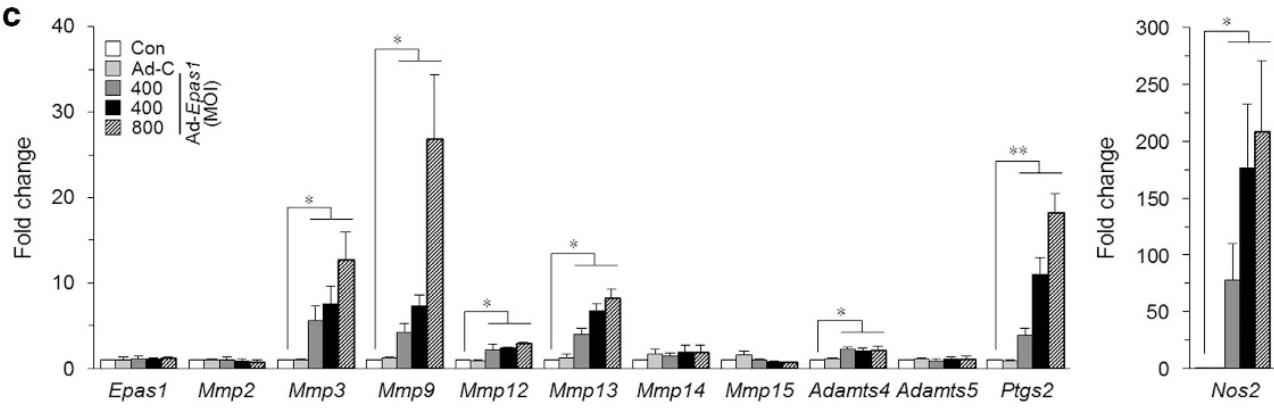

Figure 2 Altered gene expression in FLS co-cultured with HIF-2 $\alpha$-overexpressing chondrocytes. (a) Primary cultures of mouse articular chondrocytes were left untreated or infected with $800 \mathrm{MOI}$ of $\mathrm{Ad}-\mathrm{C}$ or the indicated $\mathrm{MOI}$ of Ad-Epas1 for $24 \mathrm{~h}$. The mRNA levels of the indicated genes were detected by RT-PCR analysis. ( $b$ and $\mathbf{c}$ ) Ad-Epas1-infected chondrocytes incubated on the upper chamber of a transwell plate were co-cultured with primary cultured mouse FLS for $24 \mathrm{~h}$. The expression levels of the indicated genes were determined by RT-PCR (b). Relative mRNA levels (compared to those in untreated cells) were quantified by qRT-PCR (c). Values are presented as mean \pm s.e.m. $\left({ }^{*} P<0.005,{ }^{*} P<0.001\right)$.

a
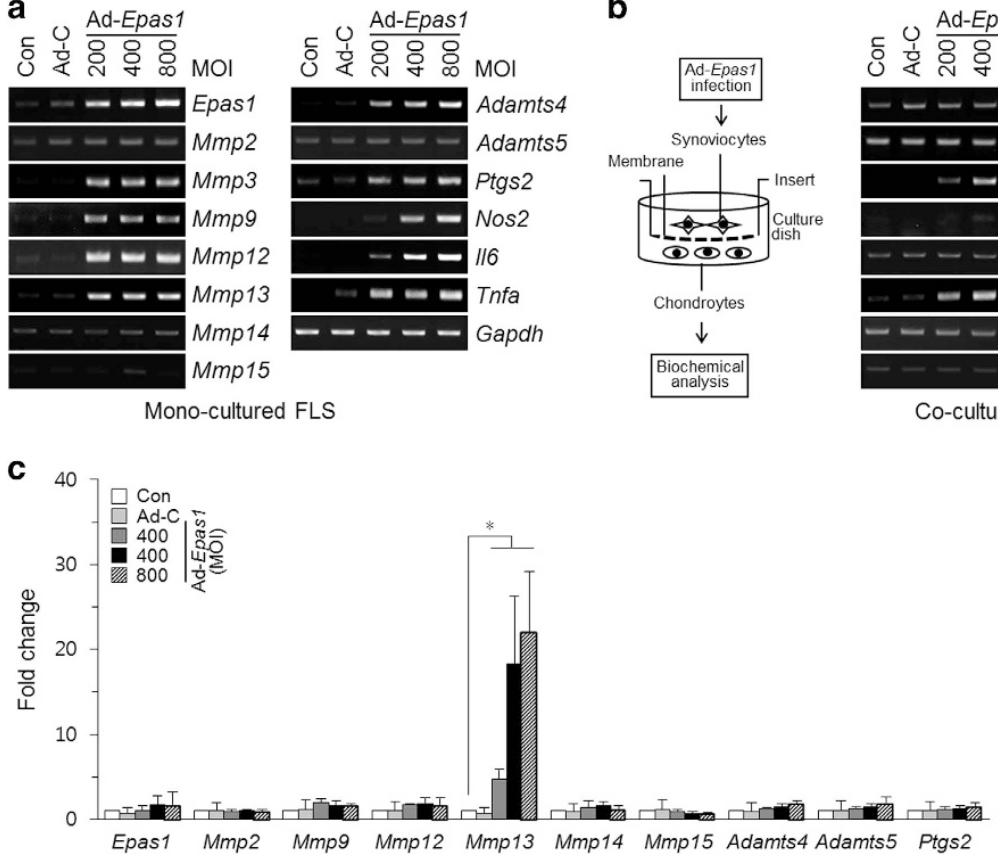

b

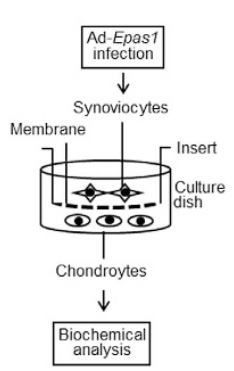

Ad-Epas1

రำ

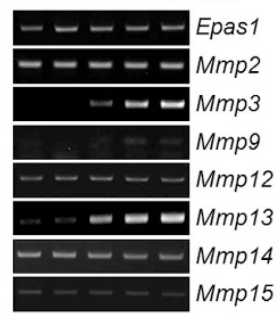

Co-cultured chondrocytes w/ FLS
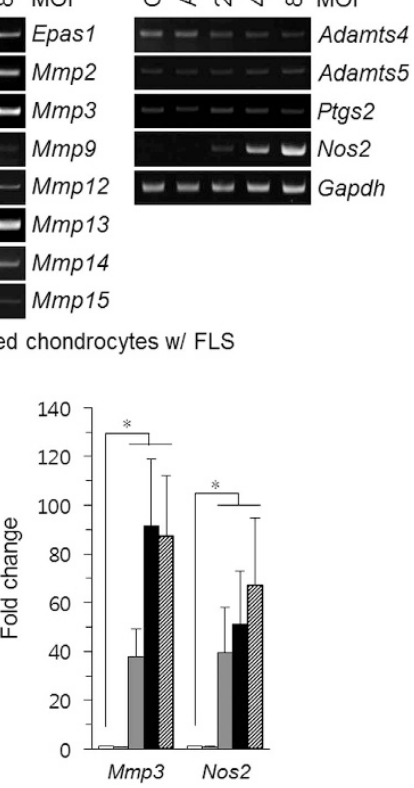

Figure 3 Altered gene expression in chondrocytes co-cultured with HIF-2 $\alpha$-overexpressing FLS. (a) Mouse FLS were left untreated or infected with $800 \mathrm{MOI}$ of Ad-C or the indicated $\mathrm{MOI}$ of Ad-Epas1 for $24 \mathrm{~h}$. The mRNA levels of the indicated genes were detected by RT-PCR. ( $b$ and $\mathbf{c}$ ) FLS were incubated in the upper chamber of a transwell plate and infected with $800 \mathrm{MOI}$ of Ad-C or the indicated amounts (MOI) of Ad-Epas1, and then incubated with mouse chondrocytes for an additional $24 \mathrm{~h}$. The expression levels of the indicated genes were determined by RT-PCR (b) and quantified by qRT-PCR (c). Values are presented as mean \pm s.e.m. $\left({ }^{*} P<0.005\right)$. 
examined by RT-PCR (Figure 3b) and quantified by qRT-PCR (Figure 3c).

\section{IL- 6 and TNF- $\alpha$ govern the crosstalk between FLS and chondrocytes}

Based on the above results, we speculated that IL- 6 and TNF- $\alpha$, which are well-known regulators of catabolic factors during arthritis, might regulate the expressional changes of matrixdegrading enzymes and inflammatory mediators in our co-culture system. In mono-cultures of FLS and chondrocytes, HIF- $2 \alpha$ overexpression remarkably induced the levels of IL-6 and TNF- $\alpha$ at the mRNA (Figures $2 \mathrm{a}$ and $3 \mathrm{a}$ ) and protein levels (Figures $4 \mathrm{a}$ and $\mathrm{b}$ ). Treatment of cells with IL-6 specifically upregulated Mmp3 and Mmp13 in FLS (Figure 4c) and chondrocytes (Figure 4d), whereas treatment with TNF- $\alpha$ induced Mmp3, Mmp9, Mmp12, Mmp13, Adamts4, Ptgs2 and Nos2 in FLS (Figure 4e) and chondrocytes (Figure 4f). These gene expression patterns were similar to those obtained in our co-culture system (Figures 2 and 3), with the effect of IL-6 treatment resembling that seen in chondrocytes co-cultured with HIF-2 $\alpha$-overexpressing FLS and the effect of TNF- $\alpha$ treatment resembling that seen in FLS co-cultured with HIF-2 $\alpha$-expressing chondrocytes. To confirm these observations and examine whether the observed co-culture-induced increases in gene expression were dependent on IL-6, we used cells isolated from Il6-knockout mice. Interestingly, $M m p 3$ and Mmp13 were upregulated to control levels in FLS co-cultured with HIF-2 $\alpha$-overexpressing IL-6-deficient chondrocytes (Figure 5a), but this upregulation was markedly reduced in chondrocytes co-cultured with HIF-2 $\alpha$-overexpressing Il6-knockout FLS (Figure 5b). These results support the idea that the HIF- $2 \alpha$-mediated upregulation of IL- 6 in FLS has an essential role in regulating catabolism-promoting gene expression in chondrocytes. Next, we examined the effects of antibody-mediated blockade of TNF- $\alpha$ signaling in our co-culture system. Treatment of HIF-2 $\alpha$-overexpressing chondrocytes with a TNF- $\alpha$-neutralizing antibody blocked the upregulations of Mmp3, Mmp9, Mmp12, Mmp13, Adamts4, ptgs 2 and Nos 2 in co-cultured FLS (Figure 6a). In contrast, blockade of TNF- $\alpha$ signaling in HIF-2 $\alpha$-overexpressing FLS did
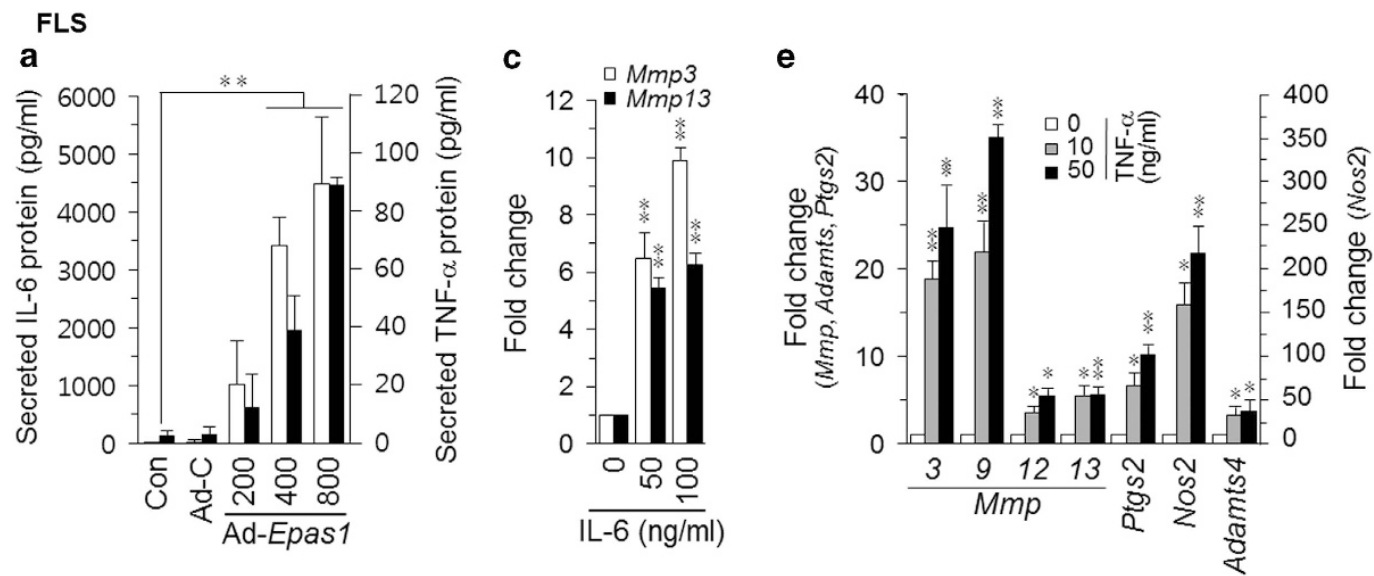

\section{Chondrocytes}

b

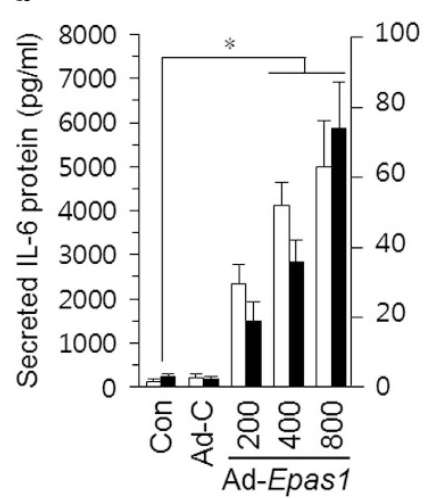

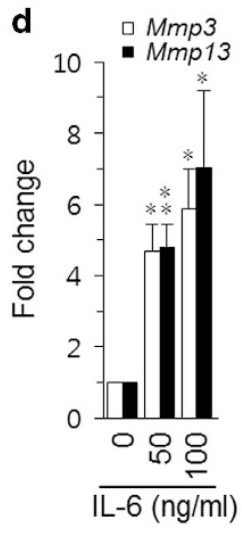

f

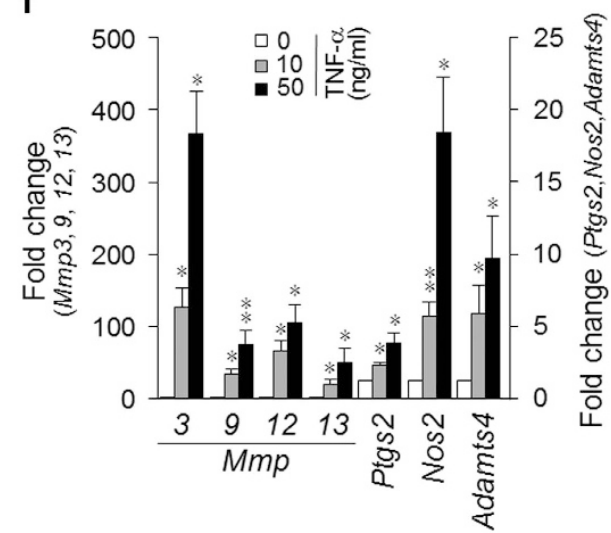

Figure 4 IL- 6 and TNF- $\alpha$ have distinct effects on FLS and chondrocytes. (a and $\mathbf{b}$ ) Mouse FLS (a) and chondrocytes (b) were infected with the indicated $\mathrm{MOI}$ of Ad-Epas1, and the levels of secreted IL-6 and TNF- $\alpha$ in the culture media were determined by ELISA. (c and d) The indicated amounts of recombinant mouse IL-6 protein were used to treat FLS (c) or chondrocytes (d) for $24 \mathrm{~h}$, and the mRNA levels of Mmp3 and Mmp13 were detected by qRT-PCR. (e and f) Mouse FLS (e) and articular chondrocytes (f) were treated with the indicated doses of recombinant mouse TNF- $\alpha$ protein for $24 \mathrm{~h}$, and the levels of the indicated catabolic factors were determined by qRT-PCR. Values are presented as means \pm s.e.m. $\left({ }^{*} P<0.01,{ }^{* *} P<0.005\right)$. 

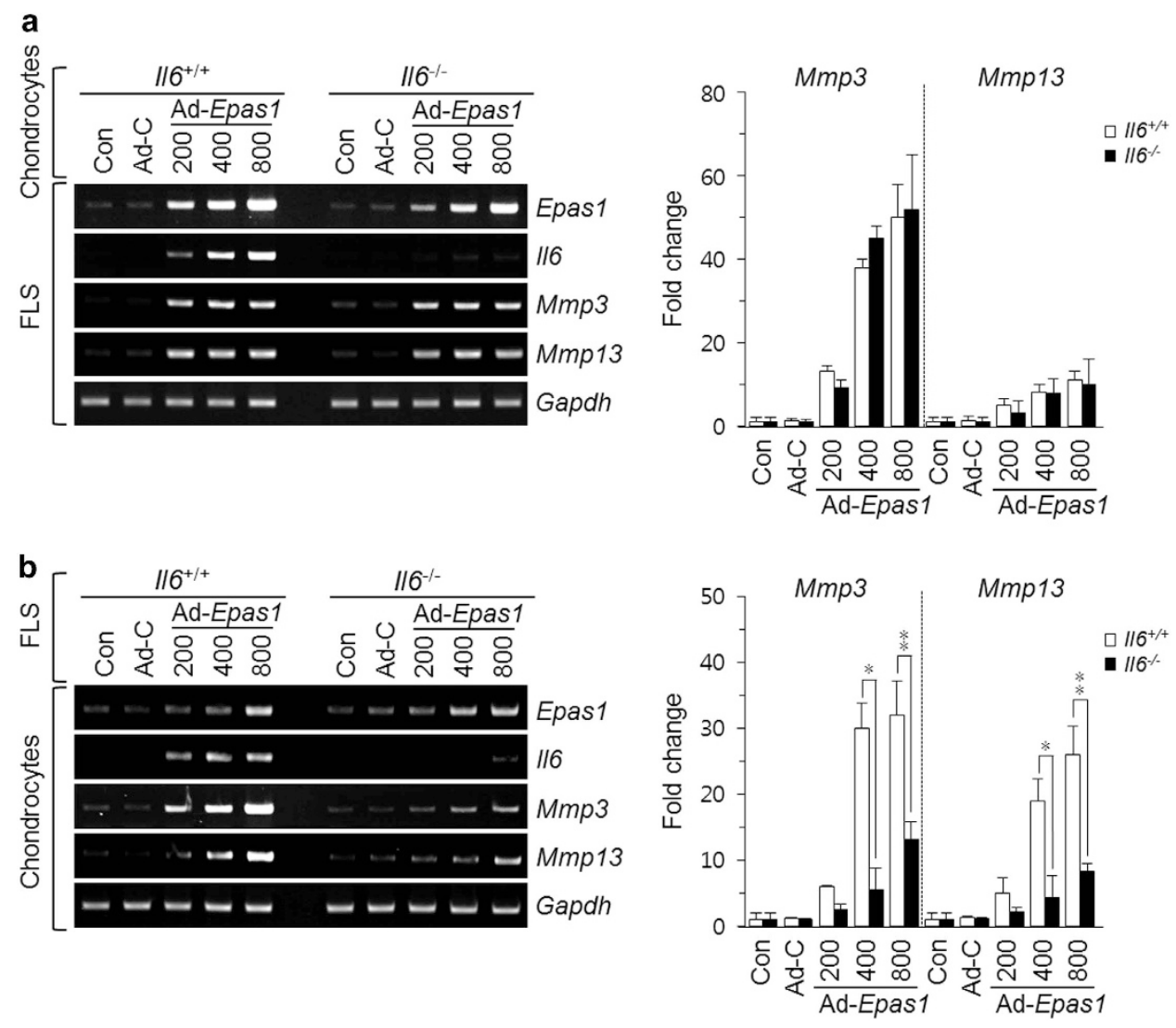

Figure 5 Blockade of IL-6 secretion in FLS or chondrocytes in co-culture. (a) Chondrocytes isolated from WT or $/ 16^{-1-}$ mice were infected with Ad-Epas 1 and co-cultured with FLS for an additional $24 \mathrm{~h}$. The expression levels of Epas1, I/6, Mmp3, and Mmp13 were detected by RT-PCR and quantified by qRT-PCR. (b) FLS isolated from WT or $1 / 6^{-/-}$mice were infected with the indicated amounts (MOI) of Ad-Epas 1 and then co-cultured with chondrocytes. RT-PCR and qRT-PCR were carried out to detect Epas1, $1 / 6, \mathrm{Mmp3}$, and Mmp13 in chondrocytes. Values are presented as means \pm s.e.m. $\left({ }^{*} P<0.05,{ }^{*} P<0.005\right)$.

not significantly inhibit the upregulations of $M m p 3, M m p 13$ and Nos2 in co-cultured chondrocytes (Figure 6b). This suggests that HIF-2 $\alpha$-induced signaling from chondrocytes to FLS (but not the reverse) depends on TNF- $\alpha$. To further examine why these effects differed even though IL- 6 and TNF$\alpha$ had the same effects on both cell types in mono-culture, we examined the expression levels of IL-6 receptors (Il6ra and Ilost) and TNF- $\alpha$ receptors (Tnfrsfla and Tnfrsfl $b$ ) in FLS and chondrocytes overexpressing HIF- $2 \alpha$. We found that the expression of Tnfrsflb was significantly increased in HIF- $2 \alpha-$ overexpressing FLS (Figure 7a), whereas that of Il6ra was significantly upregulated in HIF-2 $\alpha$-overexpressing chondrocytes (Figure 7b).

Taken together, our results suggest that HIF- $2 \alpha$ mediates the crosstalk between FLS and chondrocytes by potentiating IL-6 and TNF- $\alpha$ signaling; FLS-secreted IL-6 is a major regulator of Mmp3 and Mmp13 gene expression in chondrocytes; and chondrocyte-secreted TNF- $\alpha$ is a key regulator of Mmps, Ptgs 2 and Nos2 in FLS.

\section{DISCUSSION}

As the major target tissues in the pathogeneses of RA and OA are the synovium and cartilage, respectively, most of the previous studies on the pathology of arthritis have focus on a single-cell type, such as FLS in RA synovium or chondrocytes in OA cartilage. However, these diseases are associated with widespread damage throughout the joint. Thus, crosstalk between chondrocytes and FLS may be a crucial event in arthritis. We recently reported that HIF- $2 \alpha$ serves as an essential catabolic regulator in the pathogeneses of RA and OA by upregulating tissue-destroying genes. ${ }^{10,11}$ Here, we used an in vitro co-culture system to characterize some major mediators governing the interaction between FLS and chondrocytes in response to HIF- $2 \alpha$. As shown schematically in Figure 7c, chondrocyte-derived TNF- $\alpha$ upregulates the expression of catabolic factors in FLS, whereas FLS-derived IL-6 has an important role in regulating $M m p 3$ and $M m p 13$ in chondrocytes.

Previous studies of RA have focused on the functions and interactions of the three most abundant cell populations (FLS, macrophage-like synoviocytes and $\mathrm{T}$ lymphocytes) and other critically important immune cells (B-lymphocytes, plasma cells, dendritic cells and mast cells) in the RA synovium. ${ }^{17-19}$ At the inflammatory site, the synovial membrane becomes thickened due to the invasion of immune cells and the increased proliferation of resident $\mathrm{FLS}^{20}$ The degree of synovial 

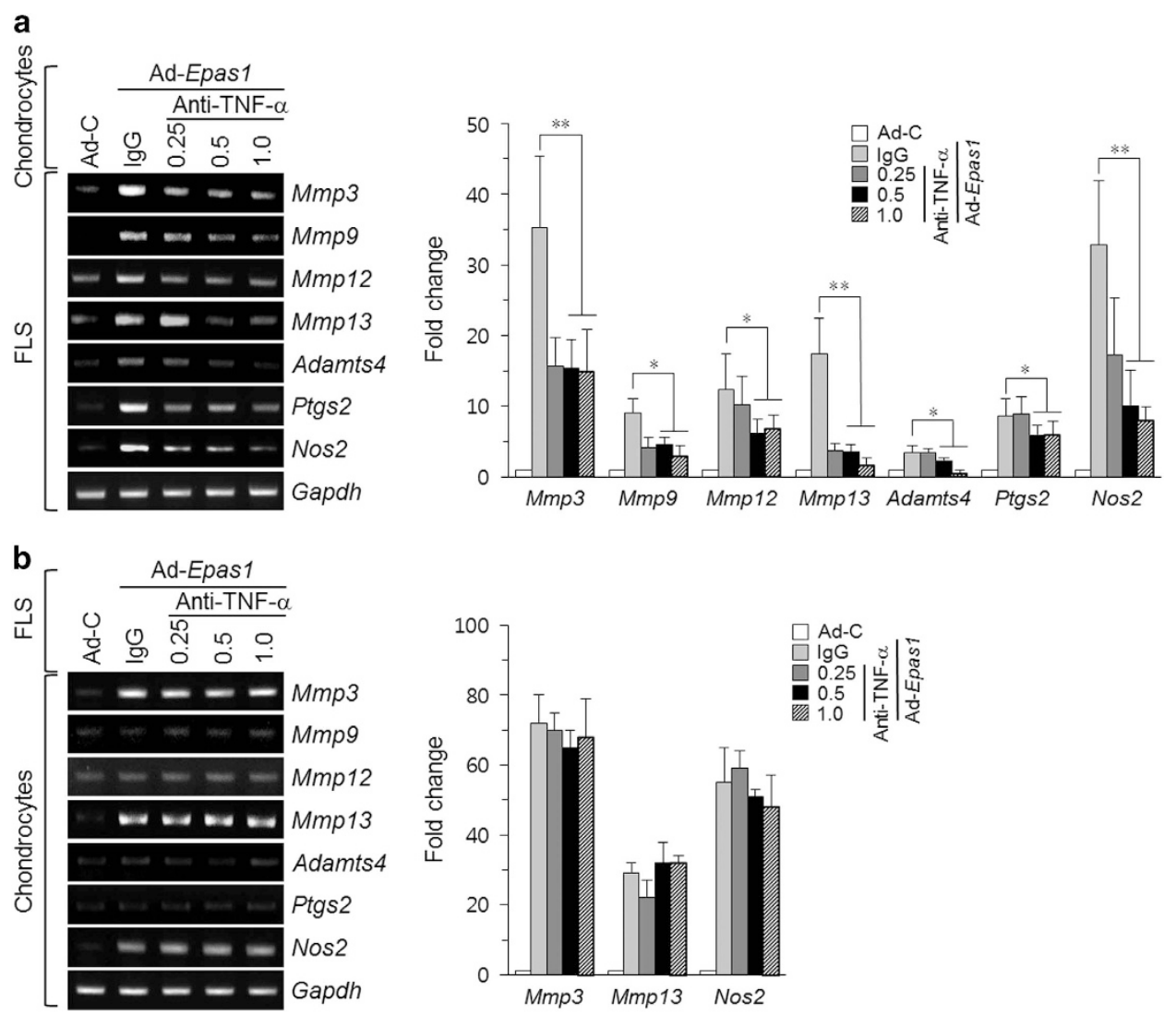

Figure 6 Blockade of TNF- $\alpha$ secretion in co-cultured FLS or chondrocytes. Mouse chondrocytes or FLS were infected with or without 800 $\mathrm{MOI}$ of Ad-C or Ad-Epas1 and incubated with FLS (a) or chondrocytes (b), respectively. After $12 \mathrm{~h}$, the indicated amounts ( $\mu \mathrm{g})$ of TNF- $\alpha$ neutralizing antibody were added. After an additional $12 \mathrm{~h}$, the transcripts of the indicated catabolic factors were detected by RT-PCR and qRT-PCR. Values are presented as means \pm s.e.m. $\left({ }^{*} P<0.05,{ }^{*} P<0.005\right)$.
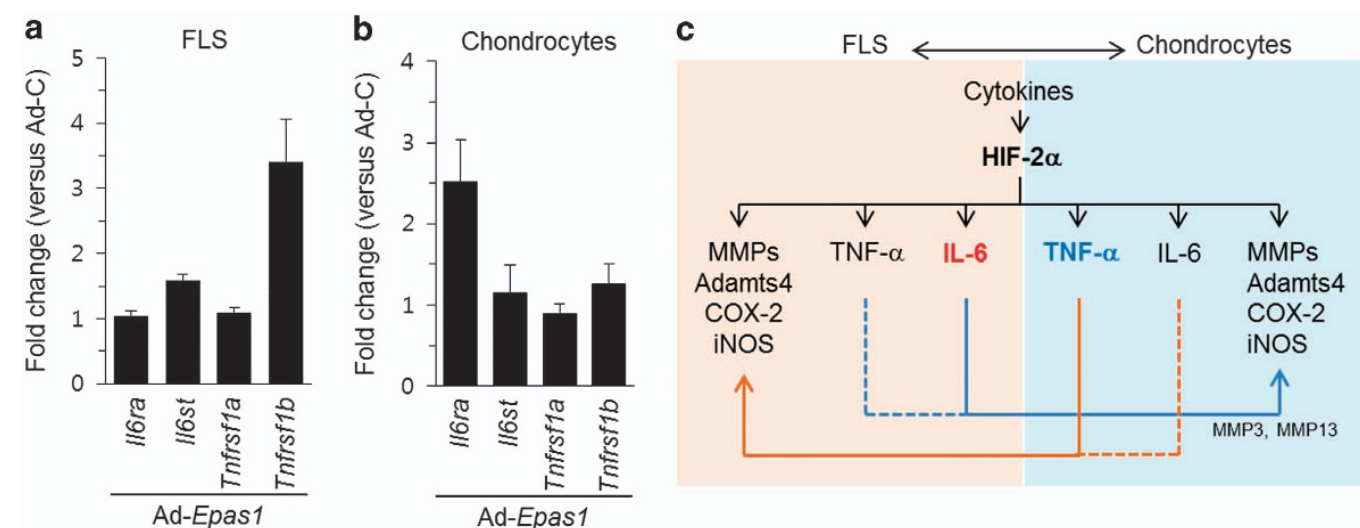

Figure 7 The receptors for IL- 6 and TNF- $\alpha$ show distinct expression patterns in HIF-2 $\alpha$-overexpressing FLS and chondrocytes. (a and $\mathbf{b}$ ) Mouse FLS or chondrocytes were infected with Ad-Epas1 or empty virus at an $\mathrm{MOI}$ of 800 for $24 \mathrm{~h}$, and microarray analyses were performed on chondrocytes and FLS. Microarray data for IL- 6 receptors ( $/ / 6 r a$ and $/ / 6 s t)$ and TNF- $\alpha$ receptors (Tnfrsfla and Tnfrsf1b) in Ad-Epas1-infected FLS (a) and chondrocytes (b) are shown. Transcript expression levels are shown relative to those in Ad-C-treated cells. (c) Schematic diagram summarizing the regulatory mechanism governing the in vitro interaction between FLS and chondrocytes in the presence of HIF-2 $\alpha$ overexpression.

hyperplasia correlates with the severity of cartilage erosion, resulting in the formation of an invasive pannus. ${ }^{21}$ FLS are main effector cells in the progression of RA, contributing to pannus formation, cartilage erosion and progressive joint destruction by secreting diverse cytokines, chemokines and matrix metalloproteinases. ${ }^{20}$ During the formation of the pannus at the synovium-cartilage interface, the migration and invasion of activated FLS into the cartilage are critical 
events. Unlike RA, OA was long considered a noninflammatory disease, largely because it affects the cartilage, which lacks blood vessels or nerves. However, it is now widely accepted that synovial inflammation is a characteristic feature of OA, and the development and progression of OA are closely related to inflammatory responses even during the early stages of the disease. ${ }^{22}$ Moreover, recent experimental data have shown that $\mathrm{OA}$ is much more complex than originally believed, ${ }^{23}$ as it involves inflammatory mediators originating from the cartilage, bone ${ }^{24}$ and synovium. ${ }^{5}$ Indeed, we found that the severity of experimental OA seems to correlate with synovial hyperplasia (Figure 1), supporting the presence of synovial inflammation in the OA joint. It seems logical, therefore, that secreted inflammatory mediators are likely to be critical regulators of the disturbed anabolism and enhanced catabolism in OA-affected joints.

We previously demonstrated that HIF- $2 \alpha$ is overexpressed during synovitis and cartilage destruction, and that it acts as an essential catabolic regulator in $\mathrm{OA}^{10}$ and inflammatory $\mathrm{RA}^{11}$ by modulating the expression levels of various catabolic factors (for example, matrix metalloproteinases) and inflammatory mediators (for example, cyclooxygenase -2 and inducible nitric oxide synthase). The present study reveals that HIF-2 $\alpha$ has distinct roles in the crosstalk between two representative cell types: FLS in the synovium and chondrocytes in cartilage. When co-cultured with HIF- $2 \alpha$-overexpressing chondrocytes, FLS exhibited upregulation of matrix-degrading enzymes (Mmp3, Mmp9, Mmp12, Mmp13 and Adamts4) and inflammatory mediators (Ptgs2 and Nos2), in a pattern resembling that seen in TNF- $\alpha$-treated FLS. Moreover, chondrocytes co-cultured with HIF- $2 \alpha$-overexpressing FLS showed upregulation of matrix metalloproteinases ( $M m p 3$ and $M m p 13)$, in a pattern similar to that observed in IL-6-treated chondrocytes. Unexpectedly, Nos2 expression is also increased in the latter system (Figure 3c), suggesting the involvement of other mediators in addition to IL-6.

Excessive secretion of soluble mediators (for example, cytokines and chemokines) is known to cause synovitis and cartilage erosion in arthritis. A broad spectrum of cytokines has been detected in joint tissues and synovial fluids, and these mediators may also contribute to the joint inflammation and cartilage matrix destruction typical of RA and OA. For instance, chemokines such as CXCL12 (SDF-1) ${ }^{25}$ and CCL20 ${ }^{26}$ stimulate proinflammatory and degradative responses in cartilage. Proinflammatory cyltokines including IL-15, IL-17, IL-18, IL-21 and LIF have been also implicated in the pathophysiologies of RA and $\mathrm{OA}$, however, upregulation of these proinflammatory cytokines by HIF-2a overexpression was insignificant in chondorcytes. $^{27}$ IL- $1 \beta$, TNF- $\alpha$ and IL- 6 seem to be the main proinflammatory cytokines involved in the pathogenesis of RA and OA. HIF- $2 \alpha$ is highly upregulated in both FLS and chondrocytes following IL- $1 \beta$ treatment. ${ }^{10,11}$ As IL-6 and TNF$\alpha$ increased in response to HIF- $2 \alpha$ overexpression, we focused on two cytokines, which are produced by chondrocytes and synovial tissues and induce the production of numerous inflammatory and catabolic factors. In patients with RA or
OA, the levels of IL- 6 and TNF- $\alpha$ are elevated in the synovial fluid, synovial membrane or cartilage. ${ }^{28}$ Biological agents that inhibit TNF- $\alpha$ signaling, such as infliximab and etanercept, have shown clinical efficacy in preventing the joint damage associated with RA. ${ }^{29}$ In addition, an anti-IL-6 antibody was shown to inhibit RA in an experimental mouse model, and the humanized anti-IL-6 receptor antibody, tocilizumab, is used as an immunosuppressive drug in RA patients. ${ }^{30}$ TNF- $\alpha$ can bind to two specific receptors, TNFR1 and TNFR2, which are both expressed in RA synovium ${ }^{31}$ and OA cartilage. ${ }^{32}$ Here, we show that HIF-2 $\alpha$ upregulates TNFR2 (encoded by Tnfrsf1b) in FLS but not chondrocytes, suggesting that chondrocyte-derived TNF- $\alpha$ may critically mediate the functions of FLS. This supported by the observation that TNF- $\alpha$ blockade did not affect co-cultured chondrocytes. IL- 6 exerts its effects via a classic signaling pathway that involves the receptor complex, a membrane-bound IL-6 receptor (IL-6R), a soluble form of IL-6R (sIL-6R) and gp130. ${ }^{33}$ Synoviocytes are known to express gp130 but not IL-6R, and can therefore only respond to IL-6 in the presence of sIL-6R. ${ }^{31}$ We previously observed that chondrocytes express gp130, IL-6R, and sIL-6R. ${ }^{34}$ Here, we further show that HIF-2 $\alpha$ upregulates IL-6R in chondrocytes but not FLS, suggesting that FLS-derived IL-6 may critically mediate the functions of chondrocytes. We confirmed these distinct effects using Il6-knockout cells and a TNF- $\alpha$ blocking antibody. More specifically, we found that the gene expression changes in chondrocytes co-cultured with HIF-2 $\alpha$-overexpressing FLS were significantly abrogated by IL-6 deficiency, whereas TNF- $\alpha$ neutralization blocked the gene expression changes in FLS co-cultured with HIF-2 $\alpha$-overexpressing chondrocytes.

Although the productions of various cytokines can vary based on the severity or complexity of the disease, we here conclude that HIF- $2 \alpha$ mediates the crosstalk between FLS and chondrocytes by potentiating IL- 6 and TNF- $\alpha$ signaling; FLS-secreted IL-6 is a major regulator in chondrocytes; and chondrocyte-secreted TNF- $\alpha$ is a key regulator in FLS. Many of the current studies have focused on identifying catabolic factors responsible for the inflammatory processes involved in OA. To our knowledge, no clinical study has sought to evaluate the effect of anti-TNF- $\alpha$ or anti-IL- 6 therapies in OA. Our findings suggest that future studies are warranted to examine the potential for targeting TNF- $\alpha$ and/or IL- 6 as a therapeutic strategy against OA. Moreover, our results suggest that targeting cartilage-derived mediators might even prove useful against RA.

\section{CONFLICT OF INTEREST}

The authors declare no conflict of interest.

\section{ACKNOWLEDGEMENTS}

This work was supported by grants from the National Research Foundation of Korea (2011-0030121), the Korea Health Technology R\&D Project through the Korea Health Industry Development Institute (KHIDI), funded by the Ministry of Health \& Welfare (HI12C1606), and Chonnam National University (2013-2899). 
1 Firestein GS. Evolving concepts of rheumatoid arthritis. Nature 2003; 423: 356-361.

2 Mclnnes IB, Schett G. The Pathogenesis of Rheumatoid Arthritis. N Engl J Med 2011; 365: 2205-2219.

3 Noss EH, Brenner MB. The role and therapeutic implications of fibroblastlike synoviocytes in inflammation and cartilage erosion in rheumatoid arthritis. Immunol Rev 2008; 223: 252-270.

4 Goldring MB, Otero M. Inflammation in osteoarthritis. Curr Opin Rheumatol 2011; 23: 471-478.

5 Sellam J, Berenbaum F. The role of synovitis in pathophysiology and clinical symptoms of osteoarthritis. Nat Rev Rheumatol 2010; 6: 625-635.

6 Pozgan U, Caglic D, Rozman B, Nagase H, Turk V, Turk B. Expression and activity profiling of selected cysteine cathepsins and matrix metalloproteinases in synovial fluids from patients with rheumatoid arthritis and osteoarthritis. Biol Chem 2010; 391: 571-579.

7 Patel SA, Simon MC. Biology of hypoxia-inducible factor-2 $\alpha$ in development and disease. Cell Death Differ 2008; 15: 628-634.

8 Greer SN, Metcalf JL, Wang Y, Ohh M. The updated biology of hypoxiainducible factor. Embo J 2012; 31: 2448-2460.

9 Gaber T, Dziurla R, Tripmacher R, Burmester GR, Buttgereit F. Hypoxia inducible factor (HIF) in rheumatology: low 02! See what HIF can do!. Ann Rheum Dis 2005; 64: 971-980.

10 Yang S, Kim J, Ryu JH, Oh H, Chun CH, Kim BJ et al. Hypoxia-inducible factor-2 $\alpha$ is a catabolic regulator of osteoarthritic cartilage destruction. Nat Med 2010; 16: 687-693.

11 Ryu JH, Chae CS, Kwak JS, Oh H, Shin Y, Huh YH et al. Hypoxia-Inducible Factor-2 alpha Is an Essential Catabolic Regulator of Inflammatory Rheumatoid Arthritis. Plos Biol 2014; 12: e1001881.

12 Mclnnes IB, Schett G. Cytokines in the pathogenesis of rheumatoid arthritis. Nat Rev Immunol 2007; 7: 429-442.

13 Glasson SS, Blanchet TJ, Morris EA. The surgical destabilization of the medial meniscus (DMM) model of osteoarthritis in the 129/SvEv mouse. Osteoarthritis Cartilage 2007; 15: 1061-1069.

14 Mankin HJ, Dorfman H, Lippiello L, Zarins A. Biochemical and metabolic abnormalities in articular cartilage from osteo-arthritic human hips. J Bone Jt Surg 1971; 53: 523-537.

15 Tang W, Lu Y, Tian Q-Y, Zhang Y, Guo F-J, Liu G-Y et al. The growth factor progranulin binds to TNF receptors and is therapeutic against inflammatory arthritis in mice. Science 2011; 332: 478-484.

16 Gosset M, Berenbaum F, Thirion S, Jacques C. Primary culture and phenotyping of murine chondrocytes. Nat Protoc 2008; 3: 1253-1260.

17 Müller-Ladner U, Ospelt C, Gay S, Distler O, Pap T. Cells of the synovium in rheumatoid arthritis. Synovial fibroblasts. Arthritis Res Ther 2007; 9: 223.

18 Kinne RW, Stuhlmüller B, Burmester G-R. Cells of the synovium in rheumatoid arthritis. Macrophages. Arthritis Res Ther 2007; 9: 224.

19 Lundy SK, Sarkar S, Tesmer LA, Fox DA. Cells of the synovium in rheumatoid arthritis. T lymphocytes. Arthritis Res Ther 2007; 9: 202.

20 Bartok B, Firestein GS. Fibroblast-like synoviocytes: key effector cells in rheumatoid arthritis. Immunol Rev 2010; 233: 233-255.

21 Bottini N, Firestein GS. Duality of fibroblast-like synoviocytes in RA: passive responders and imprinted aggressors. Nat Rev Rheumatol 2013; 9 24-33.
22 Sokolove J, Lepus CM. Role of inflammation in the pathogenesis of osteoarthritis: latest findings and interpretations. Ther Adv Musculoskelet Dis 2013; 5: 77-94.

23 Man G, Mologhianu G. Osteoarthritis pathogenesis - a complex process that involves the entire joint. J Med Life 2014; 7: 37-41.

24 Lajeunesse D, Reboul P. Subchondral bone in osteoarthritis: a biologic link with articular cartilage leading to abnormal remodeling. Curr Opin Rheumatol 2003; 15: 628-633.

25 Kanbe K, Takagishi K, Chen Q. Stimulation of matrix metalloprotease 3 release from human chondrocytes by the interaction of stromal cell-derived factor 1 and CXC chemokine receptor 4. Arthritis Rheum 2002; 46 : 130-137.

26 Alaaeddine N, Antoniou J, Moussa M, Hilal G, Kreichaty G, Ghanem I et al. The chemokine CCL2O induces proinflammatory and matrix degradative responses in cartilage. Inflamm Res 2015; 64: 721-731.

27 Ryu JH, Yang S, Shin Y, Rhee J, Chun CH, Chun JS. Interleukin-6 plays an essential role in hypoxia-inducible factor $2 \alpha$-induced experimental osteoarthritic cartilage destruction in mice. Arthritis Rheum 2011; 63 2732-2743.

28 Kapoor M, Martel-Pelletier J, Lajeunesse D, Pelletier J-P, Fahmi H. Role of proinflammatory cytokines in the pathophysiology of osteoarthritis. Nat Rev Rheumatol 2011; 7: 33-42.

29 Chen Y-F, Jobanputra P, Barton P, Jowett S, Bryan S, Clark W et al. A systematic review of the effectiveness of adalimumab, etanercept and infliximab for the treatment of rheumatoid arthritis in adults and an economic evaluation of their cost-effectiveness. Health Technol Assess 2006; 10: 1-229.

30 Woodrick R, Ruderman EM. Anti-interleukin-6 therapy in rheumatoid arthritis. Bull NYU Hosp Jt Dis 2010; 68: 211-217.

31 Ida H, Aramaki T, Nakamura H, Fujikawa K, Arima K, Tamai M et al. Different expression levels of TNF receptors on the rheumatoid synovial macrophages derived from surgery and a synovectomy as detected by a new flow cytometric analysis. Cytotechnology 2009; 60: 161-164.

32 Westacott $\mathrm{Cl}$, Barakat AF, Wood L, Perry MJ, Neison P, Bisbinas I et al. Tumor necrosis factor alpha can contribute to focal loss of cartilage in osteoarthritis. Osteoarthritis Cartilage 2000; 8: 213-221.

33 Rose-John S. IL-6 Trans-Signaling via the Soluble IL-6 Receptor: importance for the Pro-Inflammatory Activities of IL-6. Int J Biol Sci 2012; 8: 1237-1247.

34 Dayer J-M, Choy E. Therapeutic targets in rheumatoid arthritis: the interleukin-6 receptor. Rheumatology 2010; 49: 15-24.

This work is licensed under a Creative Commons Attribution-NonCommercial-NoDerivs 4.0 International License. The images or other third party material in this article are included in the article's Creative Commons license, unless indicated otherwise in the credit line; if the material is not included under the Creative Commons license, users will need to obtain permission from the license holder to reproduce the material. To view a copy of this license, visit http://creativecommons.org/licenses/by-nc-nd/4.0/ 